\title{
Establishment and Characterization of an Experimental Model of Coronary Thrombotic Microembolism in Rats
}

\author{
Ye Gu, Yupeng Bai, Jie Wu, Liqun Hu, \\ and $\mathrm{Bo}$ Gao \\ From the Department of Cardiology, Puai Hospital, Huazhong \\ University of Science and Technology, Wuban, China
}

To establish a model of coronary thrombotic microembolism in rats, either automicrothrombotic particulates (CM group) or saline control (SHAM group) was injected into temporarily clamped aortas of male Sprague-Dawley rats. After automicrothrombotic particulate injection, serum c-troponin I and von Willebrand factor levels, the no-flow area as evaluated by Thioflavin $S$, myocardial leukocyte infiltration levels, myocardial expressions of tumor necrosis factor $\alpha$ and interleukin-6, the percentage of arterioles obstructed by thrombosis, and myocardial fibrosis were all significantly increased whereas cardiac function as evaluated by echocardiography and hemodynamic measurements were significantly reduced compared with the sham group. Thus, aortic automicrothrombotic particulate injection could induce coronary microembolism in rats, and this model could be of value in improving the understanding of pathophysiology of coronary microembolism. (Am J Pathol 2010, 177:1122-1130; DOI: 10.2353/ajpath.2010.090889)

Atherosclerotic plaque rupture is a key event in the pathogenesis of acute coronary syndrome and during coronary interventions. ${ }^{1}$ It does not always result in complete thrombotic occlusion of the epicardial coronary artery with subsequent impending myocardial infarction, but may in milder forms result in the embolization of atherosclerotic and thrombotic debris into the coronary microcirculation, which has recently been identified as a potential cause of no-reflow phenomenon, myocardium ischemia, and myocardial infarctlet. Atherosclerotic embolization in the microvasculature is associated with adverse prognosis. ${ }^{1-4}$ Research results derived from suitable experimental models of coronary microembolism would help clinicians to understand the underlying pathological mechanisms and to develop effective therapies of coronary microembolism induced by atherosclerotic embolization in the microvasculature. ${ }^{5}$ Until now, plastic microspheres ranging from 20 to $900 \mu \mathrm{m}$ in diameter were injected into the coronary microcirculation to establish the coronary microembolism models in dog, swine and sheep. ${ }^{6-11}$ Experimental models were also made by injecting adenosine-diphosphate or catecholamine to induce platelet aggregation in coronary microcirculation in pigs. ${ }^{12-13}$ However, injecting microspheres with unique sizing would only occlude respective vessels with comparable sizes, while thrombotic debris in patients might vary greatly in sizing and affect vessels with different diameters. Models using adenosine diphosphate or catecholamine could mimic the platelet aggregation process during coronary microthrombosis but could not reflect the influences of various embolism components of atherosclerotic plaques. Therefore, established coronary microembolism models could only partly mimic the in vivo situation of coronary microthrombosis. In the present study, we showed that coronary microembolism could be induced by intraaortic injection of automicrothrombotic particulates varying in sizing in rats. Increased myocardial inflammatory cells infiltration and fibrosis as well as reduced cardiac function were observed post intraaortic automicrothrombotic particulates injection.

\section{Materials and Methods}

\section{Animals}

Adult male Sprague-Dawley rats weighing between 250 and $350 \mathrm{~g}$ (12 weeks old) were used in the present study. Rats were randomly assigned into coronary microthrombosis (CM) and sham $(0.2 \mathrm{ml}$ saline injection, $n=36$ each) group by random number tables. Hearts were excised at 3 hours, 24 hours, and 4 weeks after injection

\footnotetext{
Accepted for publication May 6, 2010.

Address reprint requests to Professor Ye Gu, Department of Cardiology, Puai Hospital, Huazhong University of Science and Technology, HanZheng Street 473, QiaoKou District, Wuhan, 430033, Hubei Province, China. E-mail: yegu2003cn@163.com.
} 
( $n=12$ each for sham and CM), respectively, for determination of serum c-troponin I, von Willebrand factor, areas of no-flow zone, and light microscopic and transmission electron microscopic analysis. Transthoracic echocardiography and hemodynamic measurements were performed in the 4-week group before excising the hearts.

An additional 96 rats (sham $=48, \mathrm{CM}=48$ ) were used for determination of serum c-troponin I at 6 hours postinjection, Willebrand factor at 1 week post-injection, myocardial leukocyte infiltration and myocardial expression of tumor necrosis factor (TNF) $\alpha$ and interleukin (IL)-6 at 24 hours, 1 week, and 4 weeks post-injection $(n=12$ each for sham and $\mathrm{CM}$ ).

All experiments were approved by Tongji Medical College Council on Animal Care Committee of Huazhong University of Science and Technology. Animals were maintained in accordance with the Guide for the Care and Use of Laboratory Animals published by the National Institutes of Health (NIH Publication no. 86-23, revised 1985).

\section{Preparations of Automicrothrombotic Particulates}

One day before operation, $0.5 \mathrm{ml}$ of blood was obtained from the ventral tail artery of each rat ${ }^{14}$ and was allowed to clot for 1 hour, then dried overnight in a $37^{\circ} \mathrm{C}$ oven. The clotted blood was then fragmented into thrombotic particulates with a manual processor for 5 minutes. Then 5 $\mathrm{mg}$ of thrombotic particulates were dissolved in $0.2 \mathrm{ml}$ of saline, and $10 \mu \mathrm{l}$ of mixed solution was taken for particulate number count and size determination using a Neubauer hemocytometer. Examinations showed that the diameters of all particulates were less than $150 \mu \mathrm{m}$, and the diameters of most particulates $(>90 \%)$ were less than $10 \mu \mathrm{m}$. The mean count of particulates with diameters less than $10 \mu \mathrm{m}$ was $16,800,000$; with diameters between 10 and $50 \mu \mathrm{m}$ was 640,000; with diameters between 50 and $100 \mu \mathrm{m}$ was 115,200; and with diameters between 100 and $150 \mu \mathrm{m}$ was 6000 , respectively.

\section{Induction of Coronary Microthrombosis}

Rats were anesthetized with sodium pentobarbital (50 $\mathrm{mg} / \mathrm{kg}$, i.p.), placed on a heating pad $\left(36^{\circ} \mathrm{C}-37^{\circ} \mathrm{C}\right)$ in a supine position, endotracheally intubated, and connected to a rodent ventilator (Alc-V8D, Shanghai Alcott Biotech Co., Shanghai, China). Under strict aseptic conditions, the second intercostal space was exposed through parasternotomy and a microretractor was used to separate the second and third rib to get adequate exposure of the operating region. After removing the pericardium, the ascending aorta was visualized. With use of a microvascular clip, the ascending aorta was temporarily clamped and $0.2 \mathrm{ml}$ of saline containing $5 \mathrm{mg}$ of automicrothrombotic particulates or $0.2 \mathrm{ml}$ of saline was injected into the aorta of rats over 10 seconds using a tuberculin syringe with a 28-gauge needle. The mean amount of hemorrhage ranged from 0.5 to $1 \mathrm{ml}$ after injection in animals without massive hemorrhage. The intercostals, pectoralis major, and skin were sutured layer-by-layer thereafter. During the operation, animals were monitored with electrocardiogram using standard lead II through subcutaneous needle electrodes. Postoperative penicillin (800,000 IU, i.p.) was administered daily for 7 days following operation for animals in $\mathrm{CM}$ and sham groups studied at 1 and 4 weeks.

\section{Measurement of Serum c-Troponin I}

Blood $(1.0 \mathrm{ml})$ was obtained from femoral vein in each rat at 6 hours and 24 hours after the injection prior to sacrifice, and serum c-troponin I was measured by electrochemistry and immunofluorescence method, respectively (OPUS, Behring Diagnostic Inc., San Jose, CA).

\section{Determination of von Willebrand Factor}

Blood $(1.0 \mathrm{ml})$ was obtained from femoral vein in each rat at 3 hours, 24 hours, 1 week, and 4 weeks prior to sacrifice and centrifuged at $3000 \mathrm{rpm}$. Von Willebrand factor was determined by antibody in antigen-based sandwich enzyme-linked immunosorbent assay (von Willebrand factor ELISA kit, Helena Laboratories, Beaumont, TX).

\section{Evaluation the Areas of No-Flow Zone}

Three hours after injection, a single bolus ( $1 \mathrm{ml} / \mathrm{kg}$ of $4 \%$ ) Thioflavin S (Sigma Chemical Co., St. Louis, MO) was injected via the jugular vein to stain the vascular endothelium in vivo. ${ }^{15}$ Thioflavin $\mathrm{S}$, a fluorescent yellow-green dye, stains intact endothelium and serves as a marker of perfusion. After 1 minute, the hearts were removed, atriums and right ventricle were separated from left ventricle, left ventricle was placed in a cutting apparatus (JP40, Shiyuan Science Equipment Co., Shanghai), and sectioned into 10 or 11 cross-sectional slices (each $1 \mathrm{~mm}$ in thickness) along long axis. Uneven slices (1, 3, 5, 7, 9, 11) were irradiated with UV light (365 $\mathrm{nm}$ wavelength) to determine the no-flow zone (NF, area not perfused by Thioflavin S). No-flow zone was traced and analyzed using NIH image software. The ratio of NF/left ventricle area (NF/LV) from all examined slices were calculated and averaged. Even slices (2, 4, 6, 8, 10) were used for light and transmission electron microscopic analysis.

\section{Light Microscopic Analysis}

Left ventricular tissue samples from the 3-hour group were fixed in formalin solution for 24 hours, embedded in paraffin, cut into $4-\mu \mathrm{m}$ sections, and stained with hematoxylin and eosin, Carstairs, ${ }^{16}$ and hematoxylin basic fuchsin picric acid (HBFP), ${ }^{17}$ respectively. A microscope (Olympus-BX41 TF, Olympus Co., Tokyo, Japan) incorporated with an image analysis software (Image Pro-4, Media Cybernetics, Inc., Atlanta, GA) was used to observe the microthrombosis in the coronary arteriole. One 
hundred coronary arterioles with diameters less than 100 $\mu \mathrm{m}$ were randomly observed under microscope (magnification, $\times 200$ ) and the percentage of microthrombosis in these arterioles was determined. For animals studied at 4 weeks after operation, hearts were fixed in Zenker solution after hemodynamic measurements. The entire apical segment and left ventricular tissues were embedded in paraffin, sectioned at $5-\mu \mathrm{m}$ intervals, and stained with Masson's trichrome. ${ }^{18}$ Myocardial leukocyte infiltration was observed in rats 1 day and 1 week after injection in hematoxylin and eosin-stained sections. Leukocytes were counted in 10 randomly selected visual fields in each section and five sections per rat were examined.

\section{Transmission Electron Microscopic Analyses}

Transmission electron microscopic analyses was applied in hearts from animals 3 hours after injection. Left ventricular tissues for transmission electron microscopic analyses were immersed in 2.5\% glutaraldehyde for 2 hours at $4^{\circ} \mathrm{C}$, rinsed with $0.1 \mathrm{~mol} / \mathrm{L}$ phosphate buffer $(\mathrm{pH}$ 7.2), postfixed in $1 \%$ osmium tetroxide in $0.1 \mathrm{~mol} / \mathrm{L}$ phosphate buffer for 2 hours at $4^{\circ} \mathrm{C}$, dehydrated through graded ethanol, and embedded in epoxy resin. Semithin sections were stained with toluidine blue, and ultrathin sections of the areas of interest were stained with uranyl acetate and lead citrate examined by Philips CM-120 transmission electron microscopy.

\section{Immunohistochemical Analysis}

One day, 1 week, and 4 weeks after injection, apex of the left ventricle was removed for Western blot analysis (see below) and the remaining left ventricular tissue samples were fixed in formalin solution for 24 hours, embedded in paraffin, and cut into $4-\mu \mathrm{m}$ sections for immunohistochemical analysis. Tissue sections were blocked with $10 \%$ normal serum for 1 hour at $27^{\circ} \mathrm{C}$. The blocker was removed and primary antibody (TNF antibody, Santa Cruz, CA; IL-6 antibody, R \& D Systems, Inc. Minneapolis, MN) was added. The tissue sections were incubated for 15 hours at $4^{\circ} \mathrm{C}$, washed with phosphate-buffered saline, and the peroxidase block was performed with $0.5 \% \mathrm{H}_{2} \mathrm{O}_{2}$ in methanol for 30 minutes. The respective biotinylated secondary antibody was added and incubated for 1 hour at $27^{\circ} \mathrm{C}$. Following washes with PBS, the streptavidin peroxidase label (Zymed) was incubated for 10 minutes at $27^{\circ} \mathrm{C}$. The sections were washed with PBS and incubated with AEC color development substrate (Zymed) for 10 minutes at $27^{\circ} \mathrm{C}$. Sections were washed in water and counterstained with Mayer's hematoxylin.

\section{Western Blot Analysis}

The apex of left ventricular tissues were homogenized in PBS, the collected tissues were centrifuged at 10,000 $\times$ $g$ for 10 minutes at $4^{\circ} \mathrm{C}$, and $70 \mu \mathrm{g}$ of supernatant was lysed in electrophoresis buffer and boiled for 10 minutes and subjected to electrophoresis in a SDS-polyacrylamide gel. The separated blots were transferred to nitro-

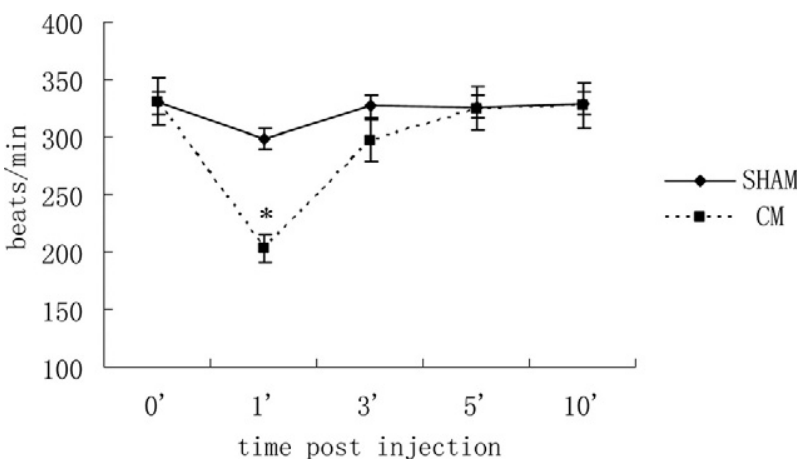

Figure 1. Heart rate changes after injection. Heart rate decreased significantly at one minute and returned to normal after five minutes in the $\mathrm{CM}$ group. Values are means $\pm \mathrm{SD} .{ }^{*} P<0.01$ versus sham group.

cellulose membranes and blocked for 1 hour in TTBS buffer containing $5 \%$ nonfat milk. The membranes were incubated overnight with primary anti-TNF $\alpha$, IL-6 polyclonal antibody (TNF $\alpha, 1: 500$ dilution; IL-6, 1:200 dilution), and incubated with a 1:1000 dilution of horseradish peroxidase-conjugated rabbit anti-goat IgG antibody for 2 hours at $37^{\circ} \mathrm{C}$. Blots were detected by chemiluminescence, and relative protein expression was quantified by scanning densitometry.

\section{Transthoracic Echocardiography}

Before operation and 4 weeks after operation, rats in the 4-week group were anesthetized (sodium pentobarbital, $50 \mathrm{mg} / \mathrm{kg}$ i.p.), lightly secured to a warming pad in the supine position, and the precordium was shaved. Transthoracic echocardiography was performed by use of a cardiac ultrasound machine with an 11.2-MHz transducer (vivid7, GE Healthcare, Piscataway, NJ). The heart was first imaged in the two-dimensional mode in the parasternal long axis and parasternal short axis views. LV areas were measured from the transverse sections and the LV short-axis lengths were used to calculate LV end systolic diameters or volumes (LVESD, LVESV) and end diastolic diameters or volumes (LVEDD, LVEDV) using the modified Simpson's rule formula. The inner endocardial mar-

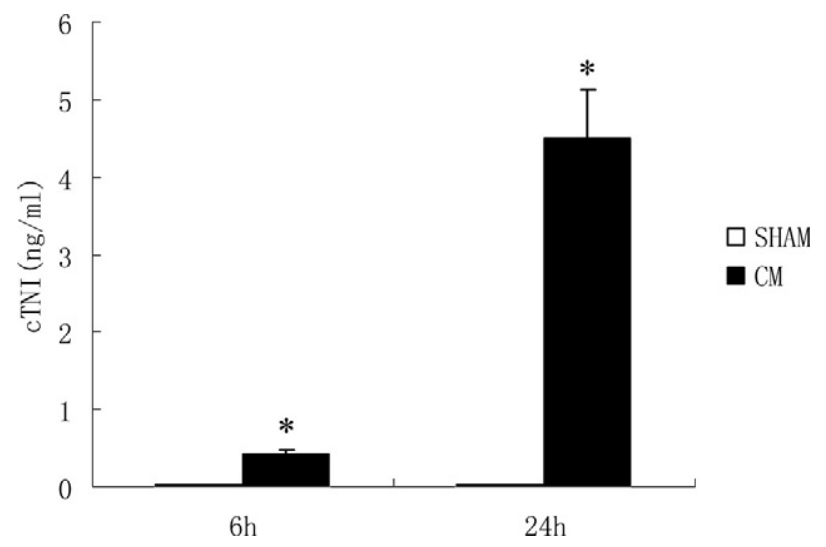

Figure 2. Serum c-TNI level after injections. Serum c-troponin I increased significantly in the CM group compared with that in the sham group. Values are means $\pm \mathrm{SD} .{ }^{*} P<0.01$ versus sham group at corresponding time post-injection. 
Table 1. Plasma vWF Level after Injection

\begin{tabular}{lcccc}
\hline Group & 3 hours & \multicolumn{1}{c}{24 hours } & 1 week & 4 weeks \\
\hline Sham & $6.05 \pm 0.22(n=10)$ & $5.92 \pm 0.12(n=10)$ & $5.94 \pm 0.12(n=10)$ & $6.08 \pm 0.16(n=10)$ \\
CM & $7.97 \pm 0.94^{\star \star}(n=9)$ & $6.66 \pm 0.54^{*}(n=8)$ & $6.02 \pm 0.32(n=8)$ & $6.23 \pm 0.15(n=7)$ \\
\hline
\end{tabular}

Values are means \pm SD.

${ }^{*} P<0.05$.

${ }^{* *} P<0.01$ versus sham group.

gin defined the LV lumen and the LVEF was derived as follows: $L V E F=(L V$ volume at end diastole $-L V$ volume at end systole)/LV volume at end diastole $\times 100 \%$. LV thickness was measured from the M-mode recording at the mid-papillary level. Results from three different cardiac cycles were averaged.

\section{Hemodynamic Measurements}

Post echocardiography measurements, rats were reanesthetized (sodium pentobarbital, 50 mg/kg i.p.), intubated, and connected to rodent respirator, LV systolic (LVSP) and end-diastolic (LVEDP), the maximum rate of rise of LV systolic pressure (dP/dtmax) and heart rate were measured via $1 \%$ heparinized short segment of fluid-filled PE 50 catheter connected to a solid-state miniature pressure transducer and the Philips apparatus (Integris Allura 12, Philips, Holland). ${ }^{19}$

\section{Statistical Analyses}

Data were expressed as mean values \pm SD. The significances of differences between groups were compared by unpaired Student's $t$-test. A value of $P<0.05$ was considered significant.

\section{Results}

\section{Pilot Study}

Before the main study, a pilot study was performed to observe the effects of automicrothrombotic particulate injection and the time course of thrombosis. Three, 5, 7, 10 , or $20 \mathrm{mg}$ of microthrombotic particulates $(n=12$ each) dissolved in $0.2 \mathrm{ml}$ of saline were injected and the mortality was $8.3 \%, 25 \%, 67 \%, 91.7 \%$, and $100 \%$, respectively. The causes of death included massive hemorrhages during injection, malignant arrhythmias, and acute cardiac failure after injection. The percentage of microthrombosis arterioles was $6.6 \pm 2.8 \%$ in the $3-\mathrm{mg}$ group and $18.4 \pm 4.8 \%$ in the 5 -mg group. Thrombosis could be evidenced at 1 hour and peaked at 3 hours and decreased at 12 hours post-injection. Therefore, $5 \mathrm{mg}$ of dried automicrothrombotic particulates dissolved in 0.2 $\mathrm{ml}$ of saline was chosen for the main study.

\section{Mortality}

In the CM group, there were eight deaths (one due to excess anesthesia, two massive hemorrhages, and five malignant arrhythmias) during the perioperative period. Another three animals died at 16 hours, 2 days, and 5 days, respectively, post-injection due to cardiac failure based on the postmortem pathological findings (pulmonary venous pleonaemia and pleural effusion). One rat without pathological evidence of microembolism was excluded from the final analysis. The remaining 24 rats were examined at 3 hours $(n=9), 24$ hours $(n=8)$, and 4 weeks $(n=7)$. In sham group, six rats died during the perioperative period (two due to excess anesthesia, three massive hemorrhages, and one malignant arrhythmia), the remaining 30 rats were examined at 3 hours $(n=10)$, 24 hours $(n=10)$, and 4 weeks $(n=10)$.

In an additional experiment for determination of myocardial leukocyte infiltration and myocardial expression of TNF $\alpha$ and IL-6, there were 12 deaths (three due to excess anesthesia, three massive hemorrhages, and six malignant arrhythmias) during the perioperative period in $\mathrm{CM}$ group, and another four animals died at 22 hours, 3 days, 4 days, and 6 days post-injection due to cardiac failure. The remaining 32 rats were examined at 6 hours $(n=9)$, 24 hours $(n=8)$, 1 week $(n=8)$, and 4 weeks $(n=7)$. In the sham group, eight rats died during the perioperative period (two due to excess anesthesia, four massive hemorrhages, and two malignant arrhythmias). The remaining 40 rats were examined at 6 hours $(n=10), 24$ hours $(n=$ 10), 1 week $(n=10)$, and 4 weeks $(n=10)$.
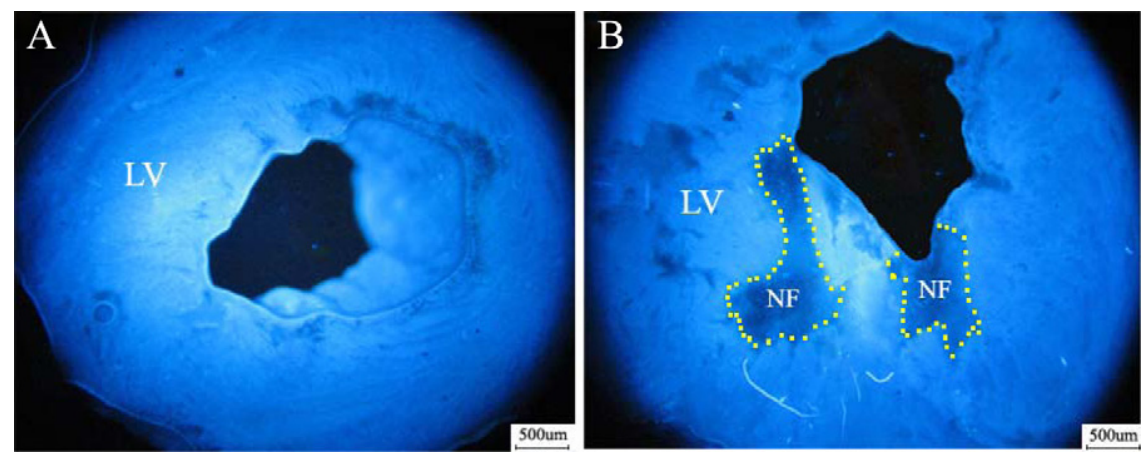

Figure 3. No-flow zone evaluation at three hours post-injection. Under UV light, the noflow zone was identified as a region deficient in fluorescence of Thioflavin S $(\mathbf{A}$, sham $\times 40 ; \mathbf{B}$ $\mathrm{CM} \times 40)$. LV indicates the left ventricle and a yellow dotted line delineates the no-flow zone. 

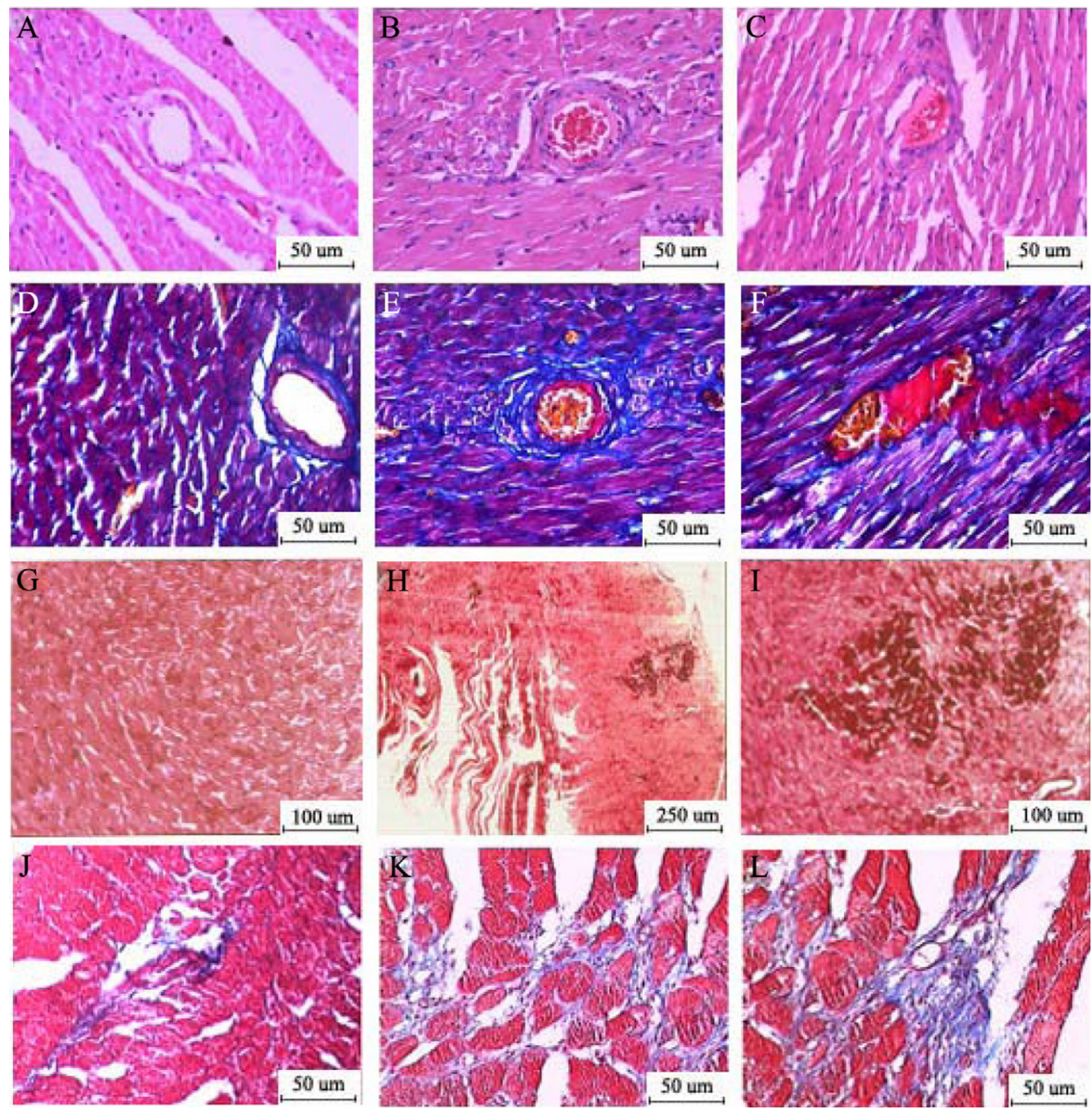

Figure 4. Light microscopic analyses. In the HE-stained slice (A, sham; $\mathbf{B}$ and $\mathbf{C}, \mathrm{CM} \times 200)$, red thrombosis was not observed in arterioles of the sham group (A) but seen in the CM group $(\mathbf{B}, \mathbf{C})$. Carstairs staining $(\mathbf{D}$, sham; $\mathbf{E}$ and $\mathbf{F}, \mathrm{CM} \times 200)$ showed the major components of thrombosis in $\mathrm{CM}$ group were fibrins (bright red) and platelets (gray-blue to navy blue). HBFP staining (G, sham; H, CM $\times 40$; I, CM $\times 100)$ showed two small flaky cardinal red regions three hours after injection in CM group $(\mathbf{H}, \mathbf{I})$. Masson staining at four weeks post-injection ( $\mathbf{J}$, sham; $\mathbf{K}$ and $\mathbf{L}, \mathrm{CM} \times 200)$ showed increased collagen deposition in CM group (K and $\mathbf{L})$.

\section{Heart Rate Changes}

After injection, heart rate decreased significantly at 1 minute and returned to baseline level 5 minutes after injection in the CM group, whereas it remained unchanged in the sham group (Figure 1).

\section{Serum c-Troponin I at 6 and 24 Hours Post-injection}

Serum c-troponin I began to increase at 6 hours postinjection and increased more significantly at 24 hours post-injection in the $\mathrm{CM}$ group $(0.45 \pm 0.06 \mathrm{ng} / \mathrm{ml}, 6$ hours; $4.50 \pm 0.87 \mathrm{ng} / \mathrm{ml}, 24$ hours) compared those in the sham group $(0.013 \pm 0.006 \mathrm{ng} / \mathrm{ml}, 6$ hours; $0.015 \pm$ 0.007 ng/ml, 24 hours; Figure 2).

\section{Plasma von Willebrand Factor Level After Injection}

Plasma von Willebrand factor was regarded as a good indicator of endothelial dysfunction and contributed to the activation of the coagulation cascade..$^{20,21}$ Our results showed that plasma von Willebrand factor levels were significantly higher at 3 hours and 24 hours and similar at 1 week and 4 weeks post-injection in the CM groups compared with sham groups (Table 1).

\section{No-Flow Zone Evaluation at 3 Hours} Post-injection

The no-flow zone was evaluated by Thioflavin S (blue fluorescence represents perfused zone and no-fluores- 


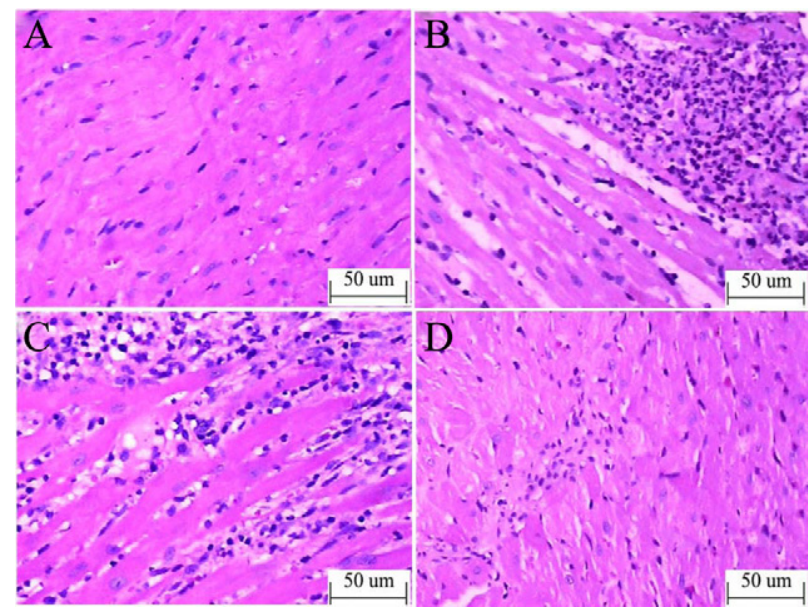

Figure 5. Inflammatory cell infiltration in HE staining. A: Sham; B: CM 24 hours; C: CM 1 week; D: CM 4 weeks $\times 200$ ) increased polymorphonuclear leukocyte infiltration in microinfarct zone in the CM group 24 hours after injection (B) and increased macrophages and leukomonocytes infiltration near microinfarct zone in CM group one week after injection $(\mathbf{C})$.

cence area represents no-flow zone examined under UV light at 365-nm wavelength). NF/LVA ratio was significantly higher in the $\mathrm{CM}$ group than that in the sham group $(10.8 \pm 3.0 \%$ versus $0.3 \pm 1.6 \%, P<0.01)$. Figure 3 , $A$ and $B$, shows representative examples of no-flow measurements.

\section{Light Microscopic Analyses}

\section{HE Staining at 3 Hours Post-injection}

Three hours after injection, red thrombus was not observed in sham group (Figure $4 \mathrm{~A}$ ) but evidenced in $18 \pm 4.53 \%$ of coronary arterioles with diameters less than $100 \mu \mathrm{m}$ in the $\mathrm{CM}$ group under a light microscope (Figure 4, A-C).

\section{Carstairs Staining at 3 Hours Post-injection}

The different ingredients of the thrombosis in arterioles showed different colors in Carstairs staining (bright red for fibrin, gray-blue to navy blue for platelets, bright blue for collagen, red for muscle, and clear yellow for red blood cells). Three hours after injection, the major components of thrombosis were fibrins and platelets and there were also red cells accumulated in the vascular lumen (Figure 4, D-F).

\section{HBFP Staining at 3 Hours Post-injection}

HBFP staining was used to detect early myocardial ischemia or infarct region. The normal cardiac muscle was stained yellow or yellow-brown, the ischemic or necrotic myocardial tissue was stained cardinal red. Ischemic area was not observed in sham rats (Figure 4G) but evidenced in $\mathrm{CM}$ rats (Figure $4, \mathrm{H}$ and $\mathrm{I}$ ).

\section{Masson Staining at 4 Weeks after Operation}

Masson staining was made in rats 4 weeks after injection. Cardiomyocytes were stained red and collagen stained blue in Masson staining. The collagen volume fraction $(C V F=$ area of the collagen/area of field of vision $\times 100 \%$ ) was measured. CVF was significantly increased in the CM group (Figure 4, K and L) compared with the sham group (Figure 4J: $5.85 \pm 1.48 \%$ versus $1.09 \pm 0.54 \%, P<0.01)$.

\section{Inflammatory Cell Infiltration}

One day after injection, coagulative necrosis occurred in the microinfarct zone, the myofibers were elongated and narrowed, and polymorphonuclear leukocyte infiltration increased in the CM group (Figure 5, A and B). One week after injection, most infiltrated leukocytes were macrophages and leukomonocytes (Figure 5C). The leukocyte counts in the CM group was significantly higher than that in the sham group at 24 hours and 1 week after the operation (Table 2). Four weeks after injection, there was no leukocyte infiltration (Figure 5D).

\section{Immunohistochemistry Staining}

One day after injection, there was only weak TNF $\alpha$ and IL-6 expression in myocardium of the sham group (Figure 6, $A$ and F). One day, 1 week, and 4 weeks after CM injection, myocardial TNF $\alpha$ expression was not only detectable at the microinfarct or peri-microinfarct zone but also evidenced at apparently "normal" zone (Figure 6, $\mathrm{B}-\mathrm{E})$, and the expression of IL-6 protein was enhanced at microinfarct and peri-microinfarct zone, vascular endothelium, and peri-vascular zone (Figure 6, G-I). Immunohistochemistry measurement also demonstrated leukocytes adhered and aggregated to vasculature (Figure 6H).

Table 2. Leukocyte Infiltration after Injection (Leukocytes $/ \mathrm{mm}^{2}$ )

\begin{tabular}{lccc}
\hline Group & 24 hours & 1 week & 4 weeks \\
\hline Sham & $162 \pm 42(n=10)$ & $168 \pm 50(n=10)$ & $152 \pm 36(n=10)$ \\
CM & $930 \pm 110^{* *}(n=8)$ & $836 \pm 130^{\star *}(n=8)$ & $158 \pm 42(n=7)$ \\
\hline
\end{tabular}

Values are means \pm SD

${ }^{*} P<0.05$.

${ }^{* *} P<0.01$ versus sham group. 

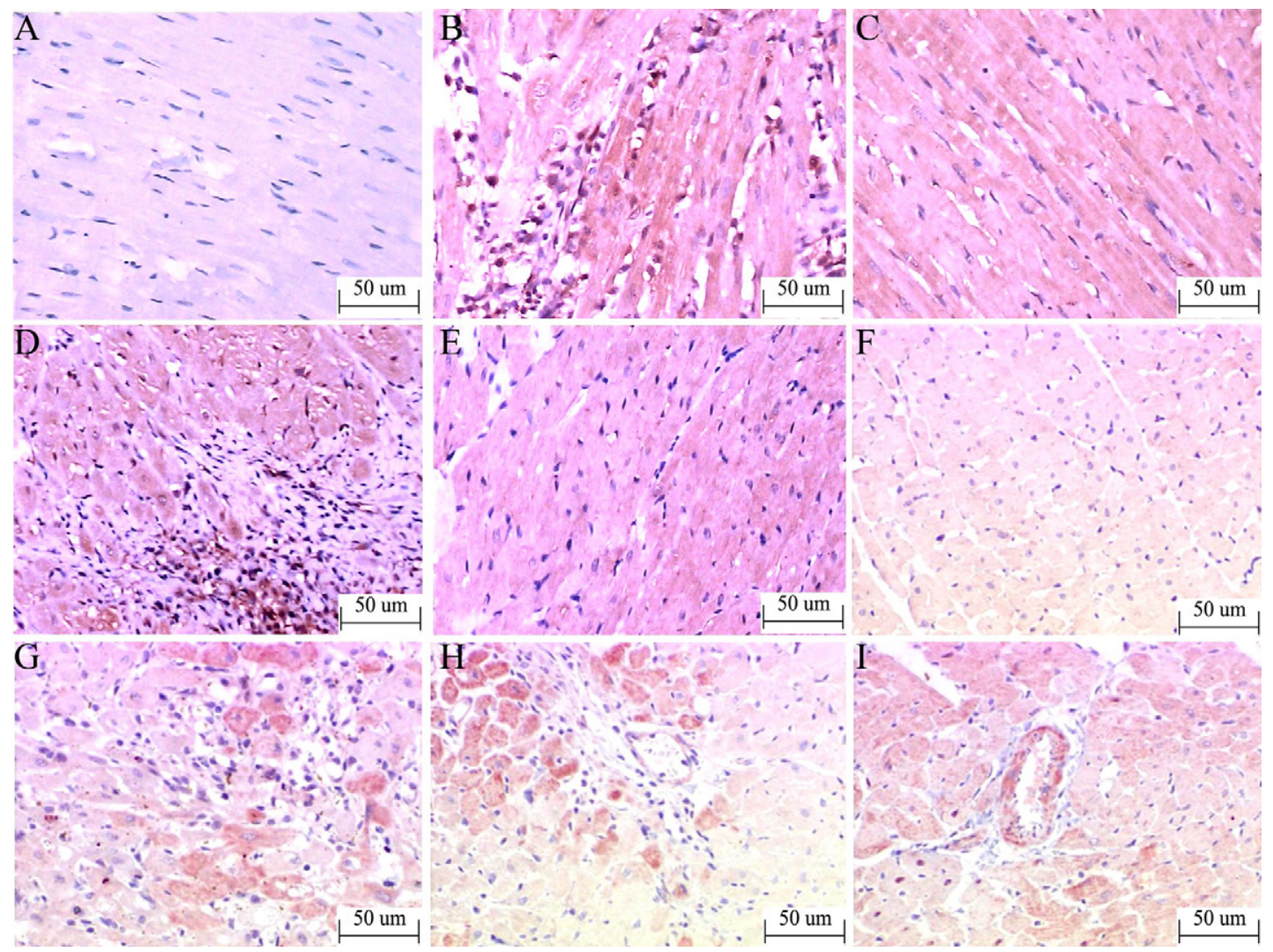

Figure 6. Immunohistochemistry staining. TNF $\alpha$ (A, sham; B, CM 24 hours at microinfarct/peri-microinfarct zone; C, CM 24 hours at "normal" myocardium; $\mathbf{D}$ CM one week at microinfarct/peri-microinfarct zone; E, CM four weeks at "normal" myocardium, $\times 200)$; and IL-6 (F, sham; G, CM 24 hours at peri-microinfarct zone; H, CM 1 week at perivascular zone; I, CM four weeks at perivascular zone, $\times 200$ ).

\section{Western Blot Analysis}

The expressions of TNF $\alpha$ and IL-6 detected by Western blotting were significantly increased in $\mathrm{CM}$ group compared with sham group at various time points after injection (Figure 7, A-C).

A
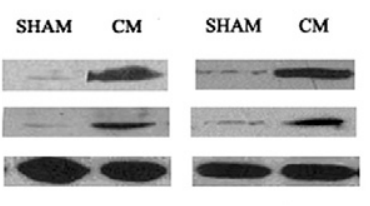

SHAM CM

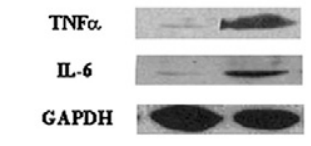

$1 d$
$1 \mathrm{w}$

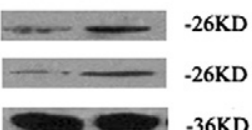

$4 w$

B

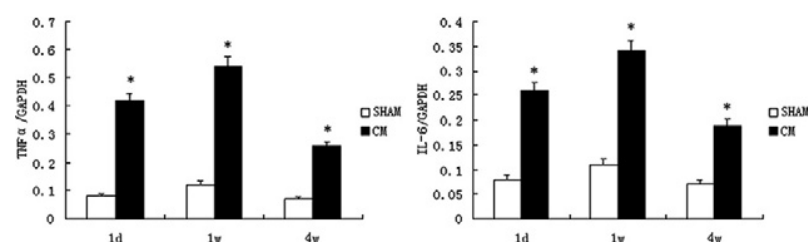

Figure 7. Western blot analyses of TNF $\alpha$ and IL-6. A: The expressions of TNF $\alpha$ and IL- 6 at different stages. B: Representative of TNF $\alpha /$ GAPDH at different stages. C: Representative of IL-6/GAPDH at different stages. Values are means $\pm \mathrm{SD} ;{ }^{*} P<0.01$ versus sham group.

\section{Electron Microscopic Analyses at 3 Hours Post-injection}

Figure $8, \quad A$ and $B$, shows myocardial ultrastructure changes (cardiomyocyte and capillary vessel) at 3 hours post-injection. Observed typical changes in the CM group were as follows: swollen and destroyed cardiomyocytes, swollen mitochondria, impaired endothelial cells and swollen basement membrane, tortuous nuclear membrane, scattered nucleoli, and condensed chromatin.
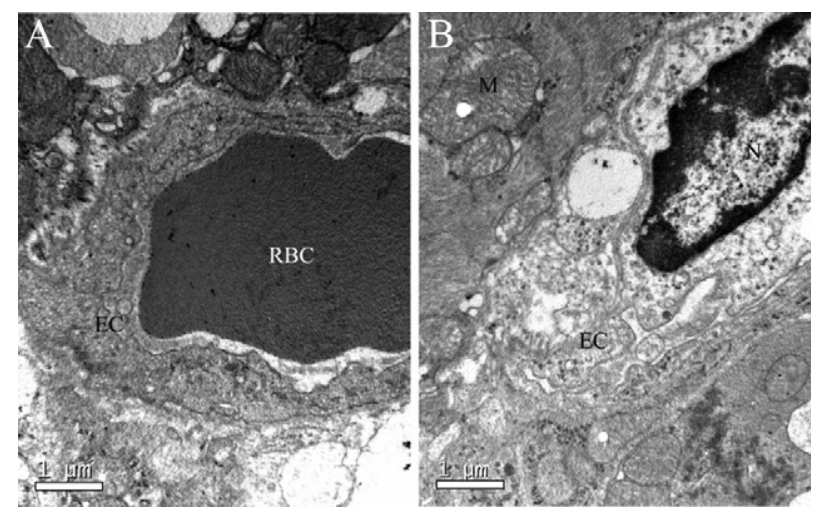

Figure 8. Electron microscopic analysis. A: Sham group. B: CM group. EC, endothelial cell; N, nucleolus; RBC, red blood cell; M, mitochondria. 
Table 3. Two-Dimensional Mode Transthoracic Echocardiography Results

\begin{tabular}{ccllllll}
\hline Group & $n$ & LVEDD $(\mathrm{mm})$ & LVESD $(\mathrm{mm})$ & LVESV $(\mathrm{ml})$ & LVEDV $(\mathrm{ml})$ & FS $(\%)$ & LVEF $(\%)$ \\
\hline Sham & & & & & & \\
$\quad$ Preoperation & 12 & $5.70 \pm 0.55$ & $2.89 \pm 0.22$ & $0.08 \pm 0.01$ & $0.51 \pm 0.06$ & $49.2 \pm 1.7$ & $84.3 \pm 5.7$ \\
$\quad$ Postoperation & 10 & $5.63 \pm 0.49$ & $2.86 \pm 0.17$ & $0.09 \pm 0.01$ & $0.51 \pm 0.05$ & $48.7 \pm 2.1$ & $82.7 \pm 6.2$ \\
CM & & & & & & \\
Preoperation & 12 & $5.74 \pm 0.49$ & $2.89 \pm 0.24$ & $0.08 \pm 0.02$ & $0.50 \pm 0.06$ & $49.7 \pm 1.4$ & $84.1 \pm 5.3$ \\
Postoperation & 7 & $6.71 \pm 0.74^{* \dagger}$ & $4.74 \pm 0.47^{* \dagger}$ & $0.39 \pm 0.12^{* \dagger}$ & $0.82 \pm 0.11^{* \dagger}$ & $28.3 \pm 5.3^{* \dagger}$ & $52.6 \pm 6.9^{* \dagger}$ \\
\hline
\end{tabular}

LVEDD, left ventricular end-diastolic dimension; LVESV, left ventricular end-systolic volume; LVEDV, left ventricular end-diastolic volume; FS fractional shortening: LVEF, left ventricular ejection fraction. Values are means \pm SD for rats.

${ }^{*} P<0.01$ versus preoperation in the same group.

${ }^{\dagger} P<0.01$ versus postoperation in sham group.

\section{Transthoracic Echocardiography}

Preoperation values were comparable between sham and CM group. Four weeks after operation, LVEDVs in the $\mathrm{CM}$ group were significantly increased, while FS and LVEF were significantly reduced compared to those in sham group (Table 3).

\section{Hemodynamical Measurements}

Left ventricular systolic pressure, the dp/dtmax and - dp/ dtmax were significantly reduced, while left ventricular end diastolic pressure was significantly increased in the CM group compared those in the sham group (Table 4).

\section{Discussion}

In this study, we showed that injection of manual processed dried automicrothrombotic particulates dissolved in saline into the aorta of male Sprague-Dawley rats when the ascending aorta was temporarily clamped successfully induced coronary microembolism. Histology examinations performed at 3 hours post-automicrothrombotic particulates injection evidenced arteriole thrombosis and ischemic regions as well as increased no-flow area. Major ingredients of the thrombosis in coronary arterioles were fibrin, aggregated platelets, and red blood cells indicating the presence of obstructed vessels by automicrothrombotic particulates and newly formed thrombosis in situ. Increased von Willebrand factor level, an indicator of endothelial function, ${ }^{22}$ at 3 hours and 24 hours post-injection in $\mathrm{CM}$ rats indicated that microthrombotic particulates also induced microvascular endothelial injury and platelet aggregation, supporting and extending prior findings by Davis et $\mathrm{al},{ }^{23}$ who showed the components of thrombus could induce endothelial dysfunction in vitro. Electron microscopic analyses evidenced impaired endothelial cells and swollen and destroyed cardiomyocytes due to microembolization and local microthrombosis. Moreover, increased serum cTnl level and myocardial leukocyte infiltration early post-injection and prolonged inflammatory responses evidenced by increased myocardial TNF $\alpha$ and IL-6 expression resembled typical inflammatory responses postischemia. ${ }^{24}$ This model therefore resembled most pathological features of coronary microembolism-induced no-reflow: coronary microembolism plus endothelial injury/dysfunction and myocardial inflammation. ${ }^{25,26}$

Acute myocardial injury was shown by increased serum c-troponin I level at 6 and 24 hours and myocardial infarctlets in HBFP-stained myocardium at 3 hours postautomicrothrombotic particulate injection in this model. In the CM group, significantly increased myocardial leukocyte infiltration was evidenced 24 hours and 1 week after injection, suggesting enhanced inflammation responses in this model. Additionally, we identified increased IL-6 expression, an important inflammatory cytokine in myocyte hypoxia after myocardial infarction and reperfused infarction, ${ }^{27,28}$ at the myocytes in peri-microinfarct zone and vascular endothelium. TNF $\alpha$ expression in the myocardium also increased not only at the microinfarct or peri-microinfarct zone but also at vascular endothelium and in apparently "normal" myocytes. In other words, coronary microembolization produced amplified myocardial inflammation through "bystander effect." This finding is in line with previous reports showing inflammatory reaction was the most important mechanism resulting in systolic heart failure in coronary microembolization and that inflammatory mediators of $\mathrm{TNF} \alpha$ play a causal role in contractile dysfunction following coronary microembolization. ${ }^{29-31}$ Increased myocardial collagen content evidenced by histology and reduced cardiac function shown by both transthoracic echocardiography and hemodynamical measurements at 4 weeks after automicrothrombotic particulates injection suggested the sequential changes in-

Table 4. Hemodynamics Measurements

\begin{tabular}{llcccc}
\hline Group & $n$ & LVSP $(\mathrm{mmHg})$ & $\operatorname{LVEDP}(\mathrm{mmHg})$ & $\mathrm{dP} / \mathrm{dtmax}(\mathrm{mmHg} / \mathrm{s})$ & $-\mathrm{dP} / \mathrm{dtmax}(\mathrm{mmHg} / \mathrm{s})$ \\
\hline Sham & 9 & $150.1 \pm 10.7$ & $5.4 \pm 3.2$ & $5616 \pm 707$ & $-5044 \pm 389$ \\
CM & 7 & $104.9 \pm 10.0^{*}$ & $18.0 \pm 3.4^{*}$ & $2979 \pm 233^{*}$ & $-2533 \pm 281^{*}$ \\
\hline
\end{tabular}

LVSP, left ventricular systolic pressure; LVEDP, left ventricular end-diastolic pressure; dP/dtmax, the maximum rate of rise of LV systolic pressure; $-\mathrm{dP} / \mathrm{dtmax}$, the maximum rate of decline of LV systolic pressure.

Values are means \pm SD for rats.

${ }^{\star} P<0.01$ versus sham group. 
duced by microembolism: coronary microembolism/coronary microthrombosis-endothelial damage/dysfunctionno reflow-infarctlets-inflammatory reaction - myocardial remodeling and cardiac dysfunction.

This model might be superior to previously reported animal models of coronary microembolization in the following respects: i) This model mimicked the in vivo situation of arteriole blockade by various sizes of microthrombi after atherosclerotic plaque rupture. ii) The components of automicrothrombotic particulates are similar as the thrombus in vivo, including fibrin, platelets, and blood corpuscle. iii) Automicrothrombotic particulates are easy to obtain and do not require elaborate equipment or large laboratories. iv) Rat model is more economical compared with large animal models. v) Rat heart is small and the entire heart could be easily sampled with few histological sections.

As a whole, this animal model exampled some of the pathological changes induced by coronary embolization seen in clinical patients with acute coronary syndromes and in patients underwent revascularization procedures (fibrinolytics or transcatheter recanalization during surgical or percutaneous procedures or prior embolization before procedures). Our model might be helpful for recognition of the pivotal role and underlying mechanism of microvascular obstruction and to test the efficacy of new therapeutic armamentarium against coronary microembolism.

\section{Acknowledgments}

We thank Dr. Kai Hu (Würzburg University, Germany) and Dr. Xinhua Gu (Fudan University, China) for their help in the preparation of this manuscript.

\section{References}

1. Skyschally A, Leineweber K, Gres P, Haude M, Erbel R, Heusch G: Coronary microembolization. Basic Res Cardiol 2006, 101:373-382

2. Erbel R, Heusch G: Coronary microembolization: its role in acute coronary syndromes and interventions. Herz 1999, 24:558-575

3. Bahrmann P, Figulla HR, Wagner M, Ferrari M, Voss A, Werner GS: Detection of coronary microembolisation by Doppler ultrasound during percutaneous coronary interventions. Heart 2005, 91:1186-1192

4. Jaffe R, Charron T, Puley G, Dick A, Strauss BH: Microvascular obstruction and the no-reflow phenomenon after percutaneous coronary intervention. Circulation 2008, 117:3152-3156

5. Topol EJ, Yadav JS: recognition of the importance of embolization in atherosclerotic vascular disease. Circulation 2000, 101:570-580

6. Weber KT, Malinin TI, Dennison BH, Fuqua JM Jr., Speaker DM, Hastings FW: Experimental myocardial ischemia and infarction: production of diffuse myocardial lesions in unanesthetized calves. Am J Cardiol 1972, 29:793-802

7. Franciosa JA, Heckel R, Limas C, Cohn JN: Progressive myocardial dysfunction associated with increased vascular resistance. Am J Physiol 1980, 239:H477-H482

8. Sabbah HN, Stein PD, Kono T, Gheorghiade M, Levine TB, Jafri S, Hawkins ET, Goldstein S: A canine model of chronic heart failure produced by multiple sequential coronary microembolizations. Am J Physiol 1991, 260:H1379-H1384

9. Ikram H, Rogers SJ, Charles CJ, Sands J, Richards AM, Bridgman
PG, Gooneratne R: An ovine model of acute myocardial infarction and chronic left ventricular dysfunction. Angiology 1997, 48:679-688

10. Gill RM, Jones BD, Corbly AK, Wang J, Braz JC, Sandusky GE, Wang $\mathrm{J}$, Shen W: Cardiac diastolic dysfunction in conscious dogs with heart failure induced by chronic coronary microembolization. Am J Physiol Heart Circ Physiol 2006, 29:H3154-H3158

11. Schmitto JD, Ortomann P, Vorkamp T, Heidrich F, Kolat P, Popov AF, Doerge H, Grossmann M, Seipelt R, Ramadori G, Schöndube A: Histological changes in a model of chronic heart failure induced by multiple sequential coronary microembolization in sheep. J Cardiovasc Surg (Torino) 2008, 49:533-537

12. Jorgensen L, Rowsell HC, Hovig T, Glynn MF, Mustard JF: Adenosine diphosphate-induced platelet aggregation and myocardial infarction in swine. Lab Invest 1967, 17:616-644

13. Haft JI, Kranz PD, Albert FJ, Fani K: Intravascular platelet aggregation in the heart induced by norepinephrine. Circulation 1972, 46:698-708

14. Brown C: Blood collection from the tail of a rat. Lab Anim (NY) 2006, 35:24-25

15. Genda S, Miura T, Miki T, Ichikawa Y, Shimamoto K: K(ATP) Channel opening is an endogenous mechanism of protection against the no-reflow phenomenon but its function is compromised by hypercholesterolemia. J Am Coll Cardiol 2002, 40:1339-1346

16. Eitzman DT, Bodary PF, Shen Y, Khairallah CG, Wild SR, Abe A, Shaffer-Hartman J, Shayman JA: Fabry disease in mice is associated with age-dependent susceptibility to vascular thrombosis. J Am Soc Nephrol 2003, 14:298-302

17. Fujita M, Fujioka Y, Ommura Y: Histopathological diagnosis of early stages of myocardial infarction: applications of the improved hematoxylin basic fuchsin picric acid (HBFP) staining method to human autopsy hearts. Hokkaido Igaku Zasshi 1985, 60:313-320

18. Goldner J: A modification of the Masson trichrome technique for routine laboratory purposes. Am J Pathol 1938, 14:237-243

19. Grossman W: Pressure measurement. Cardiac Catheterization, Angiography, and Intervention. Edited by Grossman W, Baim DS, 2000 pp. $139-141$

20. Blann AD: Plasma von Willebrand factor, thrombosis, and the endothelium: the first 30 years. Thromb Haemost 2006, 95:49-55

21. With Notø AT, Bøgeberg Mathiesen E, Amiral J, Vissac AM, Hansen JB: Endothelial dysfunction and systemic inflammation in persons with echolucent carotid plaques. Thromb Haemost 2006, 96:53-59

22. Ruggeri ZM: Von Willebrand factor, platelets and endothelial cell interactions. J Thromb Haemost 2003, 1:1335-1342

23. Davis MR, Fitzpatrick CM, Dixon PM, Kashyap VS: Thrombus-induced endothelial dysfunction: hemoglobin and fibrin decrease nitric oxide bioactivity without altering eNOS. J Surg Res 2004, 122:121-129

24. Bonvini RF, Hendiri T, Camenzind E: Inflammatory response postmyocardial infarction and reperfusion: a new therapeutic target? Eur Heart J Suppl 2005, 7:127-136

25. Reffelmann T, Kloner RA: The "no-reflow" phenomenon: basic science and clinical correlates. Heart 2002, 87:162-168

26. Rezkalla SH, Kloner RA: No-reflow phenomenon. Circulation 2002, 105:656-662

27. Deten A, Volz HC, Briest W, Zimmer HG: Cardiac cytokine expression is upregulated in the acute phase after myocardial infarction: experimental studies in rats Cardiovasc Res 2002, 55:329-340

28. Gwechenberger M, Mendoza LH, Youker KA, Frangogiannis NG, Smith CW, Michael LH, Entman ML: Cardiac myocytes produce interleukin-6 in culture and in viable border zone of reperfused infarctions. Circulation 1999, 99:546-551

29. Skyschally A, Schulz R, Haude M, Erbel R, Heusch G: Coronary microembolization: perfusion-contraction mismatch secondary to myocardial inflammation. Herz 2004, 29:777-781

30. Dörge H, Neumann T, Behrends M, Skyschally A, Schulz R, Kasper C, Erbel R, Heusch G: Perfusion-contraction mismatch with coronary microvascular obstruction: role of inflammation. Am J Physiol Heart Circ Physiol 2000, 279:H2587-H592

31. Dörge H, Schulz R, Belosjorow S, Post H, van de Sand A, Konietzka I, Frede S, Hartung T, Vinten-Johansen J, Youker KA, Entman ML, Erbel R, Heusch G: Coronary microembolization: the role of TNFalpha in contractile dysfunction. J Mol Cell Cardiol 2002, 34:51-62 